\title{
Interparticle Friction Leads to Nonmonotonic Flow Curves and Hysteresis in Viscous Suspensions
}

\author{
Hugo Perrin, ${ }^{1,2}$ Cécile Clavaud, ${ }^{2}$ Matthieu Wyart, ${ }^{1}$ Bloen Metzger, ${ }^{2}$ and Yoël Forterre ${ }^{2, *}$ \\ ${ }^{1}$ Institute of Physics, Ecole Polytechnique Fédérale de Lausanne, CH-1015 Lausanne, Switzerland \\ ${ }^{2}$ Aix Marseille University, CNRS, Institut Universitaire des Systèmes Thermiques et Industriels, \\ 13453 Marseille, France
}

(Received 11 March 2019; revised manuscript received 3 June 2019; published 16 August 2019)

\begin{abstract}
Hysteresis is a major feature of the solid-liquid transition in granular materials. This property, by allowing metastable states, can potentially yield catastrophic phenomena such as earthquakes or aerial landslides. The origin of hysteresis in granular flows is still debated. However, most mechanisms put forward so far rely on the presence of inertia at the particle level. In this paper, we study the avalanche dynamics of non-Brownian suspensions in slowly rotating drums and reveal large hysteresis of the avalanche angle even in the absence of inertia. By using microsilica particles whose interparticle friction coefficient can be turned off, we show that microscopic friction, conversely to inertia, is key to triggering hysteresis in granular suspensions. To understand this link between friction and hysteresis, we use the rotating drum as a rheometer to extract the suspension rheology close to the flow onset for both frictional and frictionless suspensions. This analysis shows that the flow rule for frictionless particles is monotonous and follows a power law of exponent $\alpha=0.37 \pm 0.05$, in close agreement with the previous theoretical prediction, $\alpha=0.35$. By contrast, the flow rule for frictional particles suggests a velocity-weakening behavior, thereby explaining the flow instability and the emergence of hysteresis. These findings show that hysteresis can also occur in particulate media without inertia, questioning the intimate nature of this phenomenon. By highlighting the role of microscopic friction, our results may be of interest in the geophysical context to understand the failure mechanism at the origin of undersea landslides.
\end{abstract}

DOI: 10.1103/PhysRevX.9.031027

\section{INTRODUCTION}

Particulate media like dry granular materials and suspensions are ubiquitous in geophysical and industrial flows [1]. Yet, understanding their flowing behavior still remains an important challenge, especially for very concentrated media where these materials can jam and exhibit a liquidto-solid transition. Until recently, dry granular materials and dense suspensions were studied separately and described with very different frameworks, as in suspensions, hydrodynamic interactions were believed to prevent physical contacts between particles [2]. However, a growing body of evidence suggests that solid contacts and interparticle friction play also a major role in dense suspensions [3-5], controlling, for instance, the packing fraction and the shear-to-normal stress ratio at which

\footnotetext{
* Corresponding author. yoel.forterre@univ-amu.fr

Published by the American Physical Society under the terms of the Creative Commons Attribution 4.0 International license. Further distribution of this work must maintain attribution to the author(s) and the published article's title, journal citation, and DOI.
}

Subject Areas: Fluid Dynamics, Interdisciplinary Physics, Soft Matter

suspensions jam [6], the scaling law of the suspension viscosity near jamming [7], or the dramatic shear thickening observed in colloidal suspensions when particles interact through an additional short-range repulsive force [8-12]. As a result, a unified description of dry granular flows and dense suspensions has emerged in recent years, based on a frictional rheology and a pressure-imposed framework $[6,7,13,14]$.

This analogy between dry granular materials and suspensions has so far mainly focused on their steady flowing behavior. Much less is known about whether such an analogy can be made for the transition between the static and the flowing regimes. A major feature of the solid-toliquid transition in dry granular materials is its hysteretic nature [15]. When a static granular material is loaded under an imposed shear stress, the level of stress required to trigger the flow is larger than the critical stress below which the flow stops. In gravity-driven flows, like heap flows or flows down inclined planes, this implies that flow starts at an angle $\theta_{\text {start }}$ larger than that at which it stops, $\theta_{\text {stop }}$ [16-18]. Since in landslides the mobilized mass is closely related to the difference between the starting and stopping angles, such hysteretical behavior plays an important role in catastrophic geophysical events. Moreover, when 
the flow starts at an angle above the stopping threshold, the avalanche quickly accelerates to a finite velocity, eventually causing catastrophic failure. Such velocity-weakening dynamics is observed in many contexts from solid friction [19] to the rupture of granular gouges [20] and large landslides [21]. Interestingly, catastrophic landslides are also observed for immersed sediments [22], where they are recognized as a potential source of tsunamis [23,24]. In such a context, it appears to be particularly important to know whether the hysteresis observed for dry granular avalanches also occurs when particles are immersed in a fluid and, through this question, to address whether here, too, the solid-to-liquid transition in dry granular flows and dense suspensions presents similarities.

The origin of hysteresis in particulate media is still under debate, but several theoretical approaches have put forward inertia as a key ingredient of the mechanism [25,26]. For a long time, this view was supported by the pioneering experiments of Courrech du Pont et al. [27]. They studied aerial and submarine granular avalanches in slowly rotating drums using different particle sizes and fluid viscosities.

For large inertia characterized by the Stokes numbers $\mathrm{St}=$ $\sqrt{\rho_{p} \Delta \rho g d^{3}} / 18 \eta$ (where $\rho_{p}$ is the particle density, $\Delta \rho=$ $\rho_{p}-\rho_{f}$ the density difference between the particles and the fluid, $d$ the particle diameter, $g$ the gravity, and $\eta$ the fluid viscosity), they reported a large and roughly constant hysteresis of the avalanche angle. However, below a critical Stokes number, hysteresis was shown to decrease as inertia decreased in the system, suggesting that no hysteresis occurs in fully overdamped suspensions.

In this article, we study the avalanche dynamics of nonBrownian granular suspensions in rotating drums for Stokes numbers much smaller than those studied in Ref. [27]. Surprisingly, we find a large hysteresis of the avalanche angle even when inertia is negligible. Using a suspension of silica microbeads where interparticle friction can be turned on or off by screening their short-range repulsive force, we show that microscopic friction is essential to trigger hysteretic avalanches. Finally, examination of the avalanche dynamics allows us to extract the effective frictional rheology of the suspensions. This analysis shows that the hysteresis observed with frictional suspensions arises from a nonmonotonic effective friction law with a velocity-weakening regime close to the flow onset. By contrast, in frictionless suspensions, the flow rule is monotonous. Overall, our work reveals the existence of hysteresis and of a velocity-weakening rheology in overdamped frictional suspensions, further unifying the flowing properties of dry granular flows and dense suspensions. Our results show that inertia is not required to observe large hysteresis in granular suspensions but that interparticle friction is key, questioning the origin of hysteresis in particulate media.

\section{RESULTS}

\section{A. Evidence of hysteresis in overdamped suspensions}

We first examine the behavior of an immersed granular pile of large glass particles $(d \approx 500 \mu \mathrm{m})$ using the classic rotating drum configuration [see Figs. 1(a) and 1(b) and the Appendix A]. By imposing a slow and constant rotation rate $\omega$, the nonbuoyant grains at the surface of the pile flow under their own weight, forming an avalanche of angle $\theta$ on top of a region experiencing a rigid rotation with the drum. At a low enough rotation rate, the avalanche dynamics is found to be unsteady, with grains being either at rest or flowing. As shown in Fig. 1(c), when the grains stand still, the pile angle $\theta$ increases at the rate $\omega$ set by the rotation rate of the drum. When the pile angle reaches $\theta_{\text {start }}$, an avalanche is spontaneously triggered, inducing a rapid downward surface flow of the grains: The pile angle decreases until it reaches $\theta_{\text {stop }}<\theta_{\text {start }}$. From there, subsequent cycles continuously repeat. The resulting sawtooth shape of the pile angle versus time is the phenomenological signature of hysteresis $[15,27]$. Its typical magnitude can be appreciated in Fig. 1(d), which shows the difference between two images taken prior to and shortly after an avalanche. Note that this hysteresis does not arise from a specific preparation protocol or precompaction of the granular pile.

The above phenomenology has been widely observed for inertial granular flows, both for aerial avalanches and for large grains immersed in low-viscosity fluids [27-31]. In agreement with Ref. [27], we find that for grains immersed in water $(\mathrm{St}=4)$, the hysteresis amplitude $\Delta \theta=\theta_{\text {start }}-\theta_{\text {stop }} \approx 1^{\circ}$ (see Fig. 5 for comparison with Courrech du Pont et al.

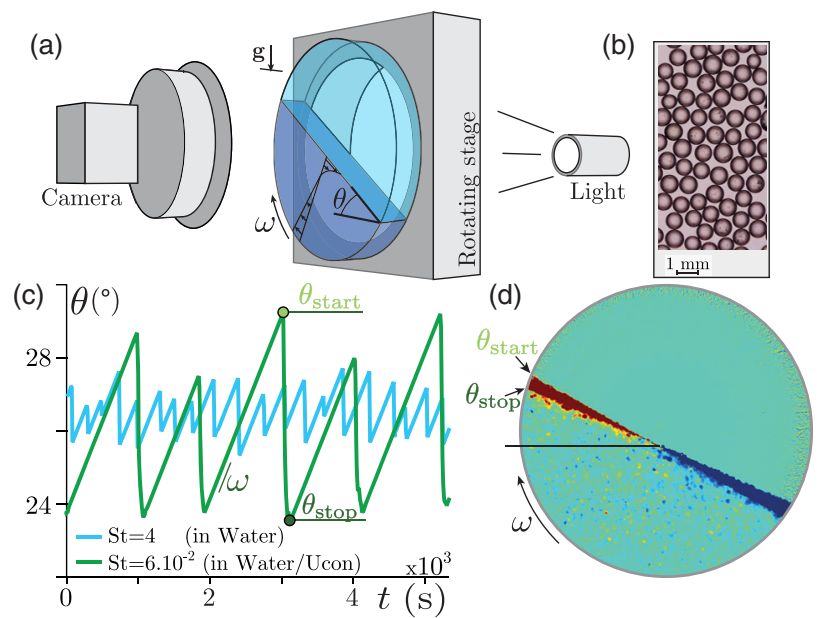

FIG. 1. Evidence of hysteresis in overdamped granular suspensions: (a) Sketch of the experimental setup. (b) Picture of the large frictional glass beads $\left(d=490 \pm 70 \mu \mathrm{m}, \rho_{p}=2500 \mathrm{~kg} \cdot \mathrm{m}^{-3}\right)$. (c) Angle of avalanche $\theta$ versus time for large glass beads immersed in pure water $(\mathrm{St}=4)$ or in a mixture of water and Ucon oil $\left(\mathrm{St}=6 \times 10^{-2}\right)$; the rotation rate of the drum is $\omega=$ $5 \times 10^{-3 \circ} \cdot \mathrm{s}^{-1}$. (d) Difference between two images taken just prior to and after an avalanche event. 

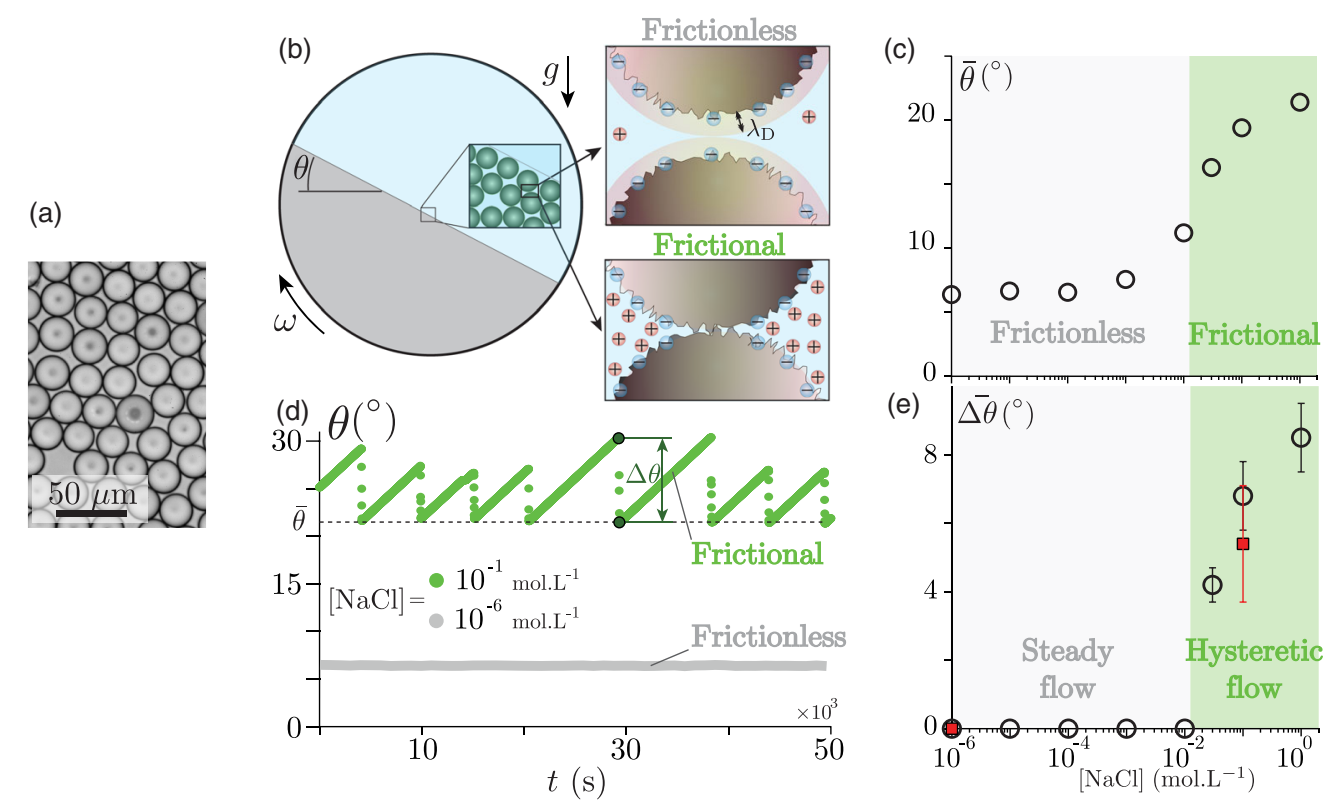

FIG. 2. Interparticle friction triggers hysteretic avalanches: (a) Picture of the silica particles ( $d=23.46 \pm 1.06 \mu \mathrm{m}$, $\rho_{p}=1850 \mathrm{~kg} \cdot \mathrm{m}^{-3}, \mathrm{St}=2 \times 10^{-2}$ ). (b) Schematic depicting how interparticle friction is tuned by varying the solvent ionic concentration. (c) Time-averaged avalanche angle $\bar{\theta}$ versus ionic concentration [ $\mathrm{NaCl}]$. (d) Avalanche angle $\theta$ versus time for frictionless (black) and frictional (green) particles. (e) Mean hysteresis amplitude $\overline{\Delta \theta}$ (averaged over 15 avalanches) versus salt concentrations. Square markers correspond to experiments performed with a water/glycerol mixture $\left(\mathrm{St}=2 \times 10^{-3}\right)$. All measurements were performed for $\omega=10^{-3}{ }^{\circ} \cdot \mathrm{s}^{-1}$ except for the water/glycerol mixture where $\omega=10^{-4} \circ \cdot \mathrm{s}^{-1}$.

data). However, in a much more viscous mixture of water and Ucon oil $\left(\mathrm{St}=6 \times 10^{-2}\right)$, the hysteresis amplitude $\Delta \theta \approx 4^{\circ}$ is significantly larger. This observation conflicts with the idea that hysteresis should vanish in fully overdamped suspensions [27]. It also suggests that the Stokes number, which compares inertia to viscous effects, is not the sole parameter that controls the amplitude of hysteretic avalanches in immersed granular media.

\section{B. Probing hysteresis by tuning microscopic friction}

Besides inertia, a key ingredient put forward in the literature to account for the emergence of hysteresis is the interparticle friction $[26,32]$. To investigate the effect of this parameter, we use a suspension composed of nonBrownian silica particles $(d \approx 24 \mu \mathrm{m})$ [Fig. 2(a)], which, as we have recently shown [10], allows the control of the interparticle friction forces (see Appendix A). When immersed in water, silica particles spontaneously develop negative surface charges, which generate an electrostatic repulsive force between the grains [33]. This force, under low confining stress (here, set by the weight of the flowing granular layer), prevents the particles from making solid contact because the range of the repulsive force, i.e., the Debye length $\lambda_{D}$, is larger than the particle roughness [Fig. 2(b), top]; in this case, the particles behave as if they were frictionless. Conversely, interparticle friction can be turned on simply by dissolving electrolytes $(\mathrm{NaCl}$ here) in water, which screens the surface charges. This method decreases $\lambda_{D}$, which eventually becomes smaller than the particle roughness [Fig. 2(b), bottom]; solid frictional contact between particles is thereby activated above a critical ionic concentration.

This transition between frictionless and frictional particles is illustrated in Fig. 2(c), showing the evolution of the quasistatic mean pile avalanche angle $\bar{\theta}$ as a function of the ionic concentration. For low salt concentration, $[\mathrm{NaCl}]<$ $10^{-3} \mathrm{~mol} \cdot \mathrm{L}^{-1}$, the mean avalanche angle is very small, $\bar{\theta} \approx 6^{\circ}$, a value remarkably close to that obtained numerically for ideal frictionless spheres, $\theta=5.76^{\circ}$ [32]. Conversely, when further increasing the salt concentration, particles start making solid contact, therefore involving friction in the avalanche dynamics: The avalanche angle increases to reach $\bar{\theta} \approx 22^{\circ}$ at large salt concentrations, a value typical for frictional grains [15,27]. As shown by Clavaud et al. [10], this transition occurs when the Debye length of the repulsive force becomes smaller than roughness of the particles.

The most important finding here is that the interparticle friction not only affects the mean avalanche angle but also the hysteretical nature of the flow [Fig. 2(d)]. When particles interact through frictional contacts (large ionic concentration $\left.[\mathrm{NaCl}]=10^{-1} \mathrm{~mol} \cdot \mathrm{L}^{-1}\right)$, one observes large hysteretic avalanches (with a sawtooth shape) similar to those obtained previously with the macroscopic beads immersed in a viscous mixture [Fig. 1(c)]. By contrast, when interparticle friction is turned off (low ionic concentration $[\mathrm{NaCl}]=$ $\left.10^{-6} \mathrm{~mol} \cdot \mathrm{L}^{-1}\right)$, hysteresis completely disappears. Grains 

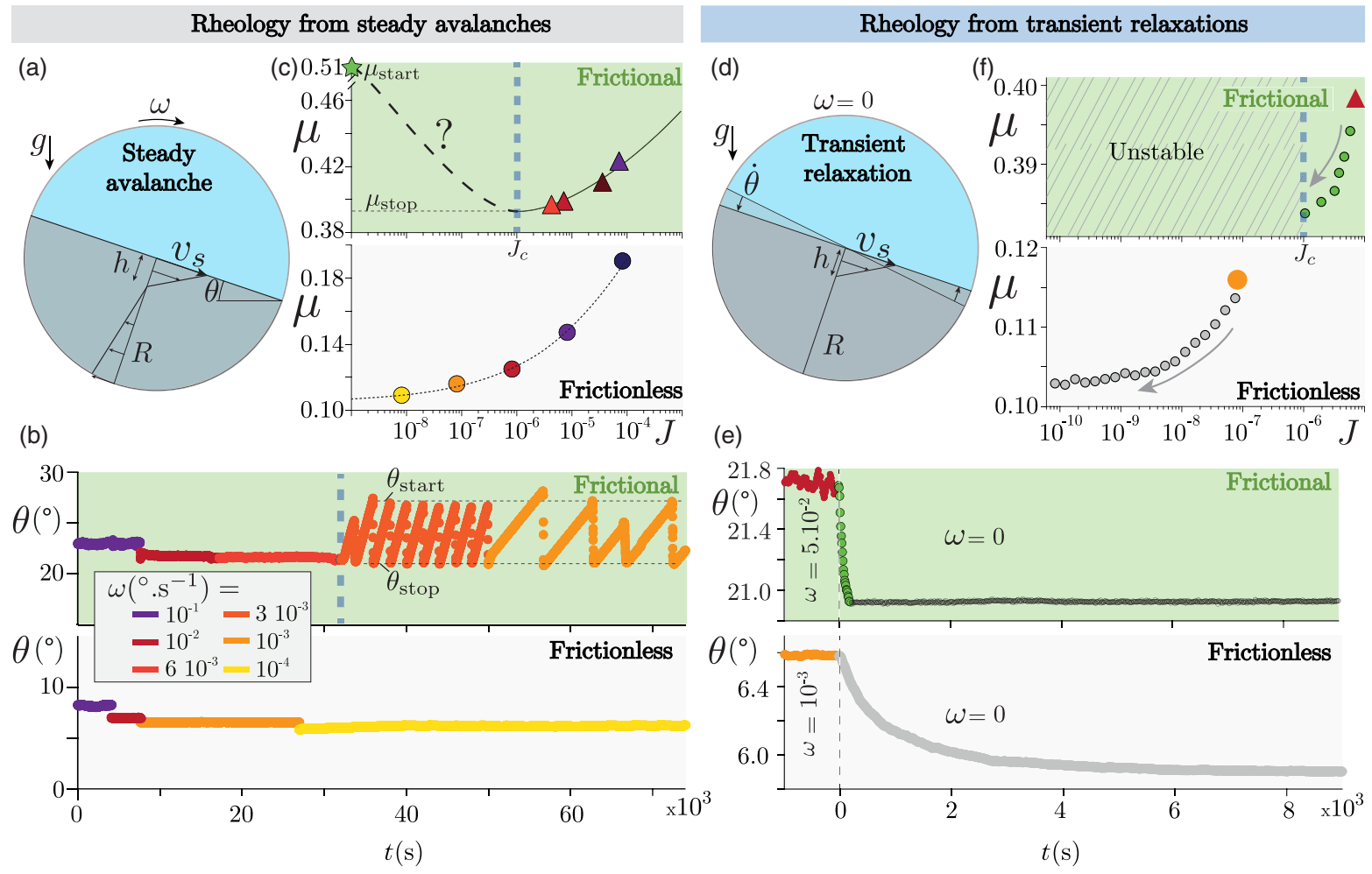

FIG. 3. Frictional and frictionless rheologies extracted from steady avalanches [(a)-(c)] and transient relaxations [(d)-(f)]. (a) Sketch defining the parameters used to extract the rheology for steady avalanches. (b) Avalanche angle $\theta$ versus time for frictional and frictionless silica particles $\left([\mathrm{NaCl}]=10^{-1}\right.$ and $10^{-6} \mathrm{~mol} \cdot \mathrm{L}^{-1}$, respectively) measured by successively decreasing the rotation rate $\omega$ of the drum. (c) Corresponding suspension effective friction coefficient $\mu$ versus viscous number $J$; the color code corresponds to the rotation rate of the drum. (d) Sketch defining the parameters used to extract the rheology from transient relaxations. (e) Relaxation of the angle of avalanche versus time for frictional and frictionless silica particles $\left([\mathrm{NaCl}]=10^{-1}\right.$ and $10^{-6} \mathrm{~mol} \cdot \mathrm{L}^{-1}$, respectively). The preparation rotation rates are $\omega=5 \times 10^{-2} \circ \cdot \mathrm{s}^{-1}$ and $\omega=10^{-3} \circ \cdot \mathrm{s}^{-1}$, respectively; the drum is stopped $(\omega=0)$ at $t=0$. (f) Corresponding $\mu$ versus $J$. The large markers correspond to the steady-state preparation, while the small markers are obtained from the relaxation where $J=\eta|\dot{\theta}| R^{2} /\left(h^{2} P\right)$ is computed from $\dot{\theta}$ measured in panel (e). The data were smoothed using time windows of variable widths, and $\dot{\theta}(t)$ was computed using the finite-difference method. The same color code as in panel (e) is used.

at the surface of the pile then flow steadily at a fixed avalanche angle. The measure of the amplitude of the hysteresis $\Delta \theta=\theta_{\text {start }}-\theta_{\text {stop }}$ for various ionic concentrations confirms that hysteresis relies on the presence of interparticle friction [Fig. 2(e)]. No hysteresis is observed as long as interparticle friction is turned off $\left([\mathrm{NaCl}]<10^{-3} \mathrm{~mol} \cdot \mathrm{L}^{-1}\right)$, while large hysteresis appears when the suspension becomes frictional $\left([\mathrm{NaCl}]>10^{-2} \mathrm{~mol} \cdot \mathrm{L}^{-1}\right)$, with an amplitude that increases with the salt concentration. We note that for the salt concentration $[\mathrm{NaCl}]=10^{-2} \mathrm{~mol} \cdot \mathrm{L}^{-1}$, no hysteresis is observed while the medium starts to become frictional, yielding an apparent discontinuity. However, as we see in the following, hysteresis can only be observed below a critical rotation rate of the drum. Here, all data were obtained at the lowest continuous rotation speed reachable with our setup. Therefore, we cannot exclude the possibility that a small hysteresis may exist at $[\mathrm{NaCl}]=10^{-2} \mathrm{~mol} \cdot \mathrm{L}^{-1}$ if we rotate the drum at lower speeds. Importantly, the Stokes number for all of these experiments is very small $\left(\mathrm{St}=2 \times 10^{-2}\right)$ and identical. Yet, the frictional suspensions exhibit hysteresis, while the frictional suspensions do not. This result confirms that inertia is not necessary to observe hysteresis in granular suspensions, while interparticle friction is (see also Fig. 5).

It is worth noting that, in our system, the emergence of hysteresis is unlikely to be attributed to adhesive forces between particles. First, for all ionic concentrations, avalanche angles have a constant slope from the top to the bottom of the avalanche, unlike adhesive powders. Second, supplementary experiments were performed in a 36\%/64\% water/glycerol mixture in order to match, down to the second digit, the index of refraction of the suspending fluid to that of the silica beads. With such an index matching, adhesive van der Waals forces are lowered by about 2 orders of magnitude compared to those expected in water [33]. Yet, the results remain similar: No hysteresis is observed at low ionic concentrations, and similar hysteresis amplitudes are found at large ionic concentrations [square markers in Fig. 2(e)]. Finally, large hysteresis is also observed using the macroscopic glass beads in the mixture 
of water and Ucon oil [Fig. 1(c)], for which adhesive forces are expected to be negligible.

\section{Link between hysteresis and rheology}

In solid friction and geophysics flows, hysteresis and stick-slip dynamics are often related to a velocityweakening regime of the flow near the onset of motion, i.e., a shear stress that decreases with the deformation rate. To understand the emergence of hysteresis in overdamped suspensions, we further analyze the dynamics of the avalanches with the aim of extracting the effective rheology of both the frictional and frictionless silica beads (immersed in solutions of ionic concentration $[\mathrm{NaCl}]=10^{-1}$ and $10^{-6} \mathrm{~mol} \cdot \mathrm{L}^{-1}$, respectively).

Under steady flow conditions, the effective rheology of the suspension can be obtained as follows. Momentum balance at the free surface implies that the macroscopic friction coefficient of the suspension $\mu$ (the ratio of the tangential to normal stresses) is directly given by the avalanche angle $\theta: \mu=\tan \theta$ [34]. Moreover, as illustrated in Fig. 3(a), conservation of mass implies that the downward flux of grains during steady avalanching is balanced by the upward motion of grains experiencing rigid rotation with the drum, yielding $h v_{s} \sim \omega R^{2}$, where $h$ is the thickness of the flowing layer, $v_{s}$ the surface velocity of the avalanche, and $R$ the radius of the drum. The typical shear rate within the flowing granular layer is $\dot{\gamma} \sim v_{s} / h$. The effective viscous number $J=\eta \dot{\gamma} / P$ characterizing the flow [6] can therefore be computed as $J=\eta \omega R^{2} /\left(h^{2} P\right)$ with $P=\phi \Delta \rho g h \cos \theta$ and the volume fraction $\phi \approx 0.6$. In these expressions, the sole unknown is the thickness of the flowing layer $h$. In the quasistatic regime of interest here, $h$ is mainly set by the grain diameter [35]. For simplicity, we use $h=50 d$ for frictionless particles and $h=25 d$ for frictional particles, as suggested by our experiments (Fig. 6).

Measuring the steady avalanche angle $\theta$ and the rotation rate $\omega$ of the drum therefore gives access to the effective frictional rheology $\mu(J)$ of the suspensions [36]. Figure 3(b) shows the steady avalanche angle obtained for various rotation rates for both frictional and frictionless particles. At large rotation rates, both systems exhibit steady flows with stationary angles that progressively decrease as the rotation rate is decreased. These data are used to extract the rheologies $\mu(J)$ in Fig. 3(c) for the frictional (top) and frictionless (bottom) grains. Each point corresponds to a different drum rotation rate [same color code as in Fig. 3(b)]. An important difference between these two systems is that below the critical rotation rate $\omega_{c}=6 \times 10^{-3 \circ} \cdot \mathrm{s}^{-1}$, the flow of frictional grains becomes hysteretic while frictionless grains flow steadily down to the smallest rotation rate accessible experimentally. This result reflects a strong difference of rheologies. Indeed, for the frictionless suspension, as the rotation rate of the drum is decreased, the effective macroscopic friction coefficient of the suspension $\mu(J)$ decreases monotonously until reaching $\mu_{0} \approx 0.1$ as
$J \rightarrow 0$ [Fig. 3(c), bottom]. This rheological flow law belongs to velocity-strengthening materials where friction grows with the deformation rate; this type of rheology yields stable flows. The behavior of frictional particles is markedly different: Below $\omega_{c}$ corresponding to a finite $J_{c}=\eta \omega_{c} R^{2} /\left(h^{2} P\right)$, the flow becomes unstable, and hysteretic avalanches come into play. In this regime, once grains are at rest, starting the flow requires the pile angle to reach $\theta_{\text {start }}$. This angle defines a starting friction coefficient $\mu_{\text {start }} \approx 0.51$ much larger than $\mu_{\text {stop }}=\mu\left(J_{c}\right) \approx 0.39$. These findings suggest that the rheology $\mu(J)$ of frictional particles is a nonmonotonous function of $J$ with a velocity-weakening region below $J_{c}$, as illustrated by the dashed line in Fig. 3(c) (top). Such multivalued rheological flow law is known to give rise to instabilities under loading, including hysteretic avalanches [37].

Steady avalanche measurements only provide the rheo$\operatorname{logy}$ for discrete values of $J$ (corresponding to the imposed rotation rates $\omega$ ). A way to obtain continuous measurements and further refine the rheology $\mu(J)$ close to the flow onset is to analyze the transient relaxations. To do so, the system is first prepared in a steady regime at a given rotation rate $\omega$ (larger than $\omega_{c}$ for frictional particles). The drum is then suddenly stopped $(\omega=0)$, and the relaxation of the pile angle $\theta(t)$ is recorded until the flow stops [Fig. 3(e)]. Assuming these relaxations are quasistationary, the macroscopic friction coefficient of the suspension $\mu$ is again given by the avalanche angle $\theta$ using the relation $\mu=\tan \theta$. Moreover, mass conservation, now that the drum is stopped $(\omega=0)$, implies that the downward grains flux $h v_{s}$ (leading to the pile angle variation $\dot{\theta}=d \theta / d t$ ) obeys $h v_{s} \sim-R^{2} \dot{\theta}$ [Fig. 3(d)]. The viscous number is then obtained as $J=\eta|\dot{\theta}| R^{2} /\left(h^{2} P\right)$.

The relaxations of frictional [Fig. 3(e), top] and frictionless [Fig. 3(e), bottom] particles are found to strongly differ both in timescale and shape. For frictionless grains, the angle of the pile relaxes slowly, asymptotically reaching its final value after about $8000 \mathrm{~s}$. Conversely, for the frictional grains, the pile angle relaxes in a much shorter time (about $50 \mathrm{~s}$ ), and its final value is reached with an abrupt change in the relaxation dynamics: The relaxation rate of the pile angle sharply transitions from a finite value to zero, yielding a sudden stop of the flow. Again, these markedly different features for the relaxations are the outcome of the intrinsically different rheological laws shown in Fig. 3(f). For frictionless grains, the rheology remains monotonous two decades below the lowest viscous number investigated with the steady-state measurements. Conversely, the rheology obtained for frictional grains confirms the existence of a critical viscous number $J_{c}$ below which no flow is possible. As the pile angle progressively relaxes, the transient avalanche stops abruptly when the system reaches the viscous number $J=J_{c}$. In Appendix B, we show that the discontinuity of $\dot{\theta}$ observed in Fig. 3(e) (top) is inherently a consequence of the finite value of $J_{c}$. 


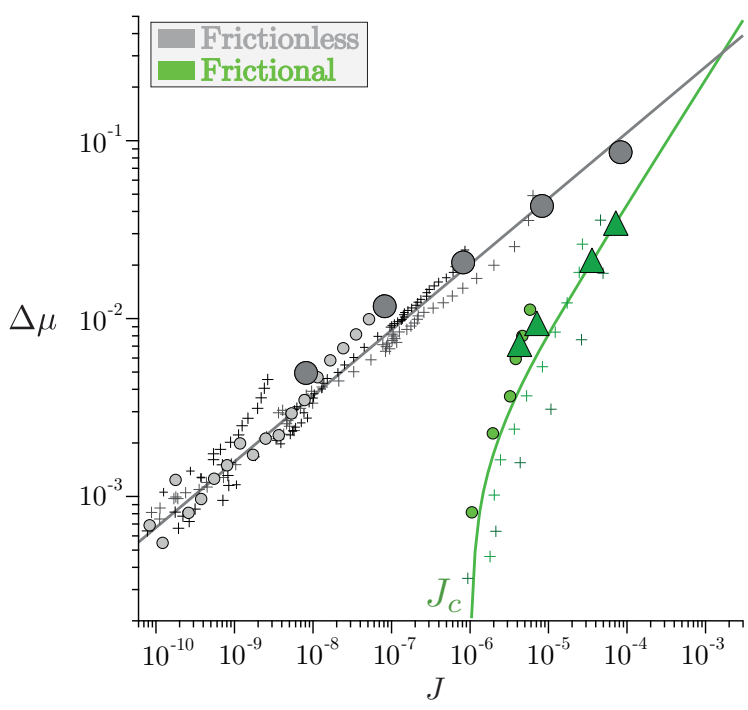

FIG. 4. Reduced macroscopic friction coefficient $\Delta \mu=\mu-\mu_{0}$ versus viscous number $J$ for frictional and frictionless particles immersed in solutions of ionic concentration $\left([\mathrm{NaCl}]=10^{-1}\right.$ and $10^{-6} \mathrm{~mol} \cdot \mathrm{L}^{-1}$, respectively). Large markers were obtained from the steady-state measurements presented in Figs. 3(a)-3(c). Small markers correspond to transient relaxations measurements from different initial steady states as described in Figs. 3(d)-3(f). Solid lines are fits (in grey) by $\Delta \mu=\left(J / J_{0}\right)^{\alpha}$, with $\alpha=0.37 \pm 0.05$ and $J_{0} \approx 2.27 \times 10^{-2}$, for frictionless particles and (in green) by $\Delta \mu=\left[\left(J-J_{c}\right) / J_{0}\right]^{\beta}$, with $\beta=0.7 \pm 0.3, J_{0} \approx 8.69 \times 10^{-3}$ and $J_{c} \approx 10^{-6}$, for frictional particles.

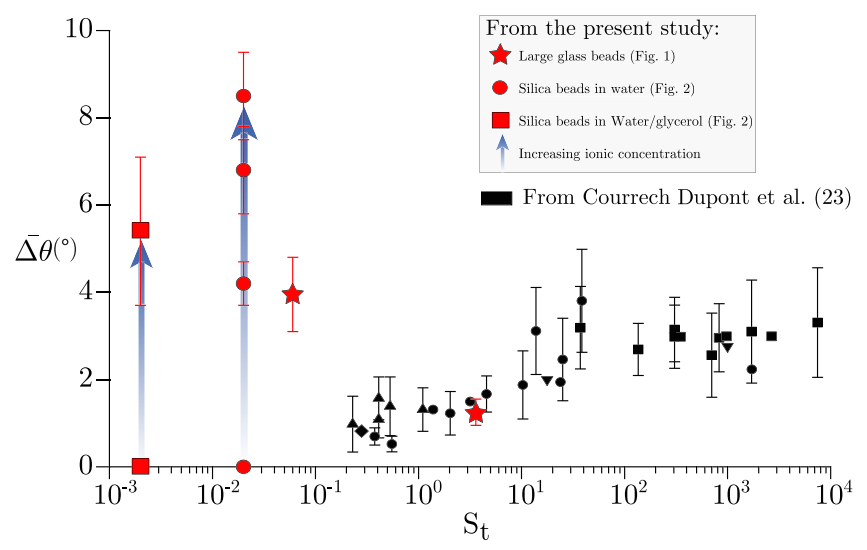

FIG. 5. Average amplitude of hysteresis $\bar{\Delta} \theta$ versus Stokes number St. We show the comparison between data obtained by Courrech du Pont et al. (23) (in black) and in the present study. Red star markers correspond to the large glass beads in water and in the mixture of Ucon-oil and water [Fig. 1(c)]. Red circle (resp. square) markers correspond to the silica particles in different salt concentration in water (resp. water/glycerol) [Fig. 2(e)]. For the present study, hysteresis amplitudes were measured at a rotation rate of $\omega=10^{-3} \circ \cdot \mathrm{s}^{-1}$, except for the silica particles in the water/glycerol mixture where $\omega=10^{-4} \circ$. $\mathrm{s}^{-1}$ and for the large glass beads where $\omega=5 \times 10^{-3 \circ} \cdot \mathrm{s}^{-1}$.
To conclude, Fig. 4 gathers the rheological laws obtained from analyzing both steady-state avalanches and transient relaxations. The reduced macroscopic friction coefficient $\Delta \mu=\mu-\mu_{0}$ is plotted versus $J$, where $\mu_{0}=\mu(J \rightarrow 0) \approx$ 0.1 for frictionless particles and $\mu_{0}=\mu_{\text {stop }} \approx 0.39$ for frictional particles [38]. The large symbols correspond to the steady-state measurements. We find that all data collapse on two separate master curves distinguishing frictionless and frictional grains. The data for frictionless grains follow the monotonous constitutive relation $\Delta \mu \sim J^{\alpha}$ with $\alpha=0.37 \pm 0.05$ over six decades of viscous number $J$. The value of the exponent is remarkably close to previous numerical observations $[7,37,39,40]$ as well as the theoretical prediction 0.35 [7]. To our knowledge, this result is the first experimental validation of these predictions. By contrast, the rheology of frictional grains exhibits a minimum at $J=J_{c}$ and, for $J>J_{c}$, can be well fitted with $\Delta \mu \sim\left(J-J_{c}\right)^{\beta}$, with $\beta=0.7 \pm 0.3$ and $J_{c} \approx 10^{-6}$.

\section{DISCUSSION}

The hysteresis observed at the onset of granular flows has generally been explained by invoking mechanisms based on inertia, such as dissipation by shocks [25,27] or endogenous acoustic noise arising from collisions [26]. In this article, by studying avalanches of viscous suspensions in rotating drums, we show that large hysteresis also occurs in overdamped systems where inertial effects are completely negligible. However, we show that the presence of interparticle friction is crucial to observe hysteresis. Avalanches of frictional particles become hysteretic below a critical rotation rate of the drum, while for frictionless particles, steady flow is always observed. Examination of the avalanche dynamics, both in the steady state and during transient relaxations, reveals the difference between the rheological laws of frictionless and frictional suspensions. Frictionless particles exhibit a monotonic behavior, while the law for frictional particles is nonmonotonic.

These rheological laws can be used to rationalize, within a simple model, the main features of the avalanches observed for both types of grains (frictionless and frictional); see Appendix C. (i) When the drum is rotated continuously, steady flows are possible only if the flow rule is stable, i.e., velocity strengthening. This condition is satisfied for all values of $J$ (or corresponding $\omega$ ) for frictionless grains but only above $J_{c}$ (or corresponding $\omega_{c}$ ) for frictional particles. Below this critical point, hysteresis emerges [Figs. 3(b) and 8(b)]. (ii) As the drum is stopped, the avalanche angle relaxes asymptotically to its final value for frictionless particles because the flow law is monotonic. By contrast, the avalanche angle for frictional particles relaxes in a finite time according to the nonmonotonic flow law [Figs. 3(e) and 8(c)]. (iii) The rheology of frictionless grains follows a power law with a lower exponent than that obtained for frictional particles $(\alpha<\beta)$. This property has a somewhat counterintuitive consequence since it implies 
that, at low viscous number, for the same friction difference $\Delta \mu$, frictionless grains must flow more slowly (smaller $J$ ) than frictional grains. This explains the long and short relaxation times observed for frictionless and frictional grains, respectively [Figs. 3(e) and 8(d)].

Our interpretation of the transition between discontinuous and continuous flow in rotating drums differs from early interpretations based on a balance between the rotation timescale and the avalanche duration [30] or on the stochastic fluctuation of the friction law [41]. Here, the critical rotation rate $\omega_{c}$ reflects the critical viscous number $J_{c}$ below which the friction law is velocity weakening and therefore unstable. Note that the rotating drum, which is a stressdriven configuration, does not give access to the unstable branch of rheology. In dry granular materials, little direct evidence of a velocity-weakening regime exists $[26,42,43]$, but several works have suggested the existence of a minimum in the friction law $[18,31,44]$. In dense suspensions, measurements and numerical simulations of the $\mu(J)$ rheology have so far reported only monotonic laws [6,45-47]. However, very few data are available for viscous numbers smaller than the critical viscous number reported here, $J_{c} \approx 10^{-6}$. An important question is to understand the physical parameters that set this value of $J_{c}$. One possibility could be that $J_{c}$ is size dependent and vanishes for large systems, as was suggested in the case of inertial grains [18]. Another possibility could be that $J_{c}$ is related to an intrinsic timescale not included in the hard sphere limit. For instance, the collision timescale introduced in the endogenous noise mechanism for inertial particles [26] could be replaced by a viscoelastic rearrangement time for overdamped systems.

Whatever the mechanism, our work shows that solid contact and interparticle friction is required to observe hysteresis in dense suspensions, as in dry materials [26,32]. Interestingly, the dilatancy exhibited by granular materials at the onset of the flow (Reynold dilatancy) is also a signature of friction between particles $[10,32]$. Thus, dilatancy and hysteresis could share the same origin, an idea already found in Bagnold's work [48]. On the other hand, solid friction is also known to involve aging phenomena and stress-weakening [12] or velocity-weakening [19] behaviors. It is thus likely that the hysteresis observed at a macroscopic level is strongly affected by the hysteresis of solid friction at the contact level. Note, however, that, in our experiment, the contact stress is fixed (set by gravity) over the entire range of $J$ investigated, ruling out the stress weakening of microscopic friction observed in Ref. [12] to explain our observations.

Overall, our study reveals that the flow onset in dense suspensions is hysteretical and that this hysteresis stems from the presence of interparticle friction. This finding provides more strong evidence that solid contacts are crucial to understanding the dynamics of suspensions close to the jamming transition, corroborating recent advances in the field [3]. In the geophysical context, our results may help us to understand the failure mechanism of undersea landslides and better predict the occurrence of massive events [24]. Interestingly, both overdamped suspensions and inertial granular materials are found to exhibit similar hysteretical signatures. Whether this hysteresis arises from collective effects or simply reflects the intrinsic hysteresis of solid friction at the scale of particle contacts is an important open question. To address this issue, it would be interesting to extend current discrete simulations of dense suspensions $[46,47]$ to account for hysteresis at the scale of particle contact.

\section{ACKNOWLEDGMENTS}

We thank O. Pouliquen and M. Trulsson for discussions, P. Dame and E. Fernandez for performing preliminary experiments, and S. Noël and F. Ratouchniack for help in building the experimental setup. M. W. and H. P. thank the Swiss National Science Foundation for support under Grant No. 200021-165509 and the Simons Foundation Grant No. 454953 (M. W.). This work was supported by the European Research Council under the European Union Horizon 2020 Research and Innovation Programme (ERC Grant Agreement No. 647384), and by the Labex MEC (ANR-10-LABX-0092) under the A*MIDEX project (ANR-11-IDEX-0001-02) funded by the French government program Investissements d'Avenir.

\section{APPENDIX A: MATERIALS AND METHODS}

\section{Particles}

The grains used in Fig. 1 are large glass beads of diameter $d=490 \pm 70 \mu \mathrm{m}$ and density $\rho_{p}=2500 \mathrm{~kg} \cdot \mathrm{m}^{-3}$. The silica beads used in Figs. 2-4 are commercial particles from Microparticles $\mathrm{GmbH}$ with diameter $d=23.46 \pm 1.06 \mu \mathrm{m}$ and density $\rho=1850 \mathrm{~kg} \cdot \mathrm{m}^{-3}$.

\section{Rotating drum experiments}

The drum used in Fig. 1 (for the large glass beads) has a diameter of $52 \mathrm{~mm}$ and a depth of $10 \mathrm{~mm}$ with a coarsened side wall and is made out of plexiglass plates. It is filled either with pure (microfiltered) water or with a mixture of Ucon oil 75-H-90,000 and water of viscosity $\eta_{f}=57 \mathrm{mPa} . \mathrm{s}$ and density $\rho_{f}=1005 \mathrm{~kg} \cdot \mathrm{m}^{-3}$. The drum used in Figs. 2-4 (for the silica particles) has a diameter of $12 \mathrm{~mm}$ and a depth of $3 \mathrm{~mm}$ with a coarsened side wall. The front and back walls are both made out of silica slides. The drum is filled with various ionic $(\mathrm{NaCl})$ aqueous solutions. Both drums are half filled with particles and fully filled with the liquid. The drums are mounted on a precision rotating stage (M-061PD from PI piezo-nano positioning). Images are acquired with a $2048 \times 1080$ Basler digital camera with a resolution of about $10 \mu \mathrm{m} / \mathrm{pix}$. The angle of avalanche is measured with a precision of $0.01^{\circ}$ using a subpixel detection of the interface between grains and liquid. 


\section{Cleaning and preparation protocol}

Prior to performing experiments, both the silica particles and the silica walls of the drum are first cleaned in piranha solution $\left(1: 2\right.$ of $\left.\mathrm{H}_{2} \mathrm{O}_{2}: \mathrm{H}_{2} \mathrm{SO}_{4}\right)$ for 10 minutes, then rinsed several times with pure microfiltered water. They are then immersed in the desired ionic solution, placed in an ultrasonic bath for 10 minutes, and rinsed 4 times with the ionic solution. After testing that the suspending fluid conductivity corresponds to the desired ionic concentration, the grains are immediately placed in the drum. Note that the amplitude of hysteresis $\Delta \theta$ can depend significantly on the cleaning and preparation protocol. However, the low-ionic-concentration water cases are less sensitive (hysteretic avalanches were never observed for $[\mathrm{NaCl}]<10^{-3} \mathrm{~mol} \cdot \mathrm{L}^{-1}$ ).

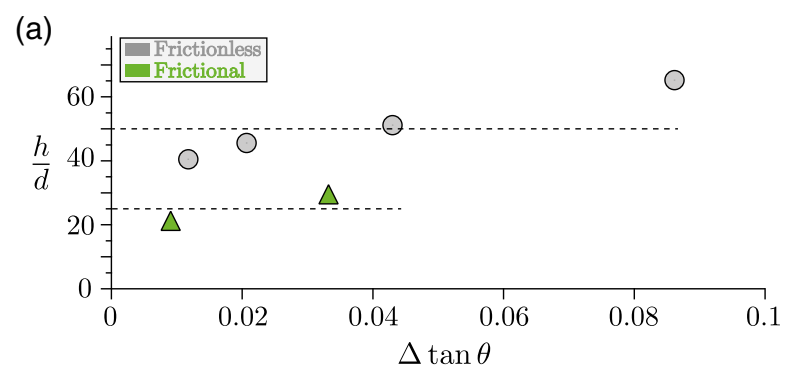

(b)

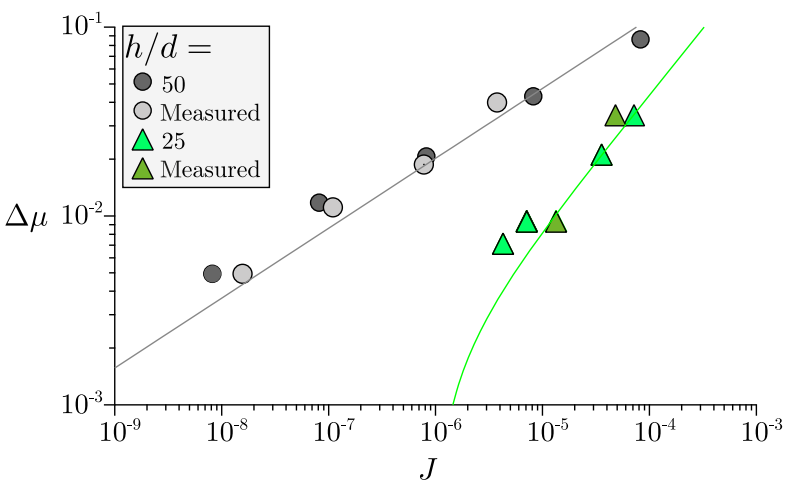

FIG. 6. (a) Flowing thickness $h / d$ of the avalanche in the steady states measured at the front wall of the drum using particle image velocimetry, as a function of the reduced avalanche angle $\Delta \tan \theta$ for frictionless (grey circles, $[\mathrm{NaCl}]=10^{-6} \mathrm{~mol} \cdot \mathrm{L}^{-1}$ ) and frictional (green triangles, $[\mathrm{NaCl}]=10^{-1} \mathrm{~mol} \cdot \mathrm{L}^{-1}$ ) particles (silica spheres of diameter $d=23.46 \pm 1.06 \mu \mathrm{m}$ and density $\left.\rho_{p}=1850 \mathrm{~kg} \cdot \mathrm{m}^{-3}\right)$. Dashed lines correspond to average values $h / d=25$ (frictional grains) and $h / d=50$ (frictionless grains). Note that the flowing depth for frictionless particles is always much smaller than the critical depth $\left(h_{c} / d=400\right)$ for which the weight of the grains overcomes the interparticle repulsive force so that contacts become frictional [10]. (b) Rheology $\mu(J)$ extracted from the stationary regimes using the averaged value of $h / d$ or the measured values shown in Fig. 3(a) for computing the viscous number $J=\eta \omega R^{2} /\left(h^{2} P\right)$ [grey symbols are frictionless grains and green symbols are frictional grains; we use the same experimental conditions as in Fig. 3(a)].

\section{APPENDIX B: COMPLEMENTARY MEASUREMENTS}

\section{Average Amplitude of Hysteresis $\overline{\Delta \theta}$ Versus Stokes Number St}

Figure 5 shows a compilation of our data and that obtained by Courrech du Pont, et al. [23].

\section{Flowing thickness measurements}

In this section, we provide complementary measurements to validate our approximations of constant flowing thickness. Figure 6(a) reports the measured flowing thickness for both frictional and frictionless silica particles in the steady states. Figure 6(b) compares the difference of rheological curves between the constant flowing thickness approximation and the consideration of the measured flowing thickness.

\section{Asymptotic friction coefficients}

Figure 7 compiles the different asymptotic values of $\mu_{0}$ and $\mu_{\text {stop }}$ obtained from steady state and relaxation measurements.

\section{APPENDIX C: SIMPLE AVALANCHE MODEL IN THE ROTATING DRUM}

Here, we derive a simple model to predict the avalanche dynamics in the rotating drum for the frictional and frictionless suspensions. For simplicity, the surface flow velocity $v_{s}$ and thickness $h$ of the avalanche are assumed to be uniform. In this case, mass conservation implies that the temporal variation of the angle of the pile of grains $\theta(t)$ is increased by the upward motion of static grains due to the solid rotation of the drum and decreased by the downward motion of grains due to the flow, that is,

$$
\frac{d \theta}{d t} \approx-\frac{h v_{s}}{R^{2}}+\omega
$$

where $R$ is the radius of the drum and $\omega$ its rotation rate. Within the quasistationary approximation, the momentum balance at the free surface of the pile implies that $\mu=\tan \theta$, where $\mu$ is the macroscopic friction coefficient of the suspension (ratio of shear to normal stress). Finally, the constitutive relation of the suspension is $\mu=\mu(J)$, where $J=\eta \dot{\gamma} / P$ is the viscous number, $\eta$ is the fluid viscosity, $\dot{\gamma} \approx v_{s} / h$ is the shear rate, and $P \approx \phi \Delta \rho g h \cos \theta$ is the pressure, with $\phi \approx 0.6$ the packing fraction, $\Delta \rho$ the particle density minus the fluid density, and $g$ the gravity. The time evolution of the avalanche angle is then given by

$$
\frac{d \theta}{d t} \approx-\frac{h^{3} \Delta \rho g \phi \cos \theta}{\eta R^{2}} \mathcal{J}(\tan \theta)+\omega,
$$

where $\mathcal{J}(\mu)$ is the reciprocal function of $\mu(J)$.

For frictionless suspensions, experiments suggest that the thickness $h=50 d$ and that the rheology is monotonous 


\begin{tabular}{|c|c|c|c|c|c|c|}
\hline & \multirow{2}{*}{$\begin{array}{l}\text { Fitting } \\
\text { steady } \\
\text { states }\end{array}$} & \multicolumn{5}{|c|}{ Fitting relaxations starting from $\omega=$} \\
\hline & & $10^{-1}$ & $5.10^{-2}$ & $10^{-2}$ & $10^{-3}$ & $10^{-4}$ \\
\hline$\mu_{0}$ & 0.104 & 0.096 & $x$ & 0.099 & 0.102 & 0.106 \\
\hline$\mu_{\text {stop }}$ & 0.389 & 0.377 & 0.374 & 0.383 & $x$ & $x$ \\
\hline
\end{tabular}

$\times$ no data available

FIG. 7. Values of $\tan \theta$ when the flow stops for frictionless (top, $[\mathrm{NaCl}]=10^{-6} \mathrm{~mol} \cdot \mathrm{L}^{-1}$ ) and frictional (bottom, $[\mathrm{NaCl}]=$ $10^{-1} \mathrm{~mol} \cdot \mathrm{L}^{-1}$ ) particles (silica spheres of diameter $d=23.46 \pm$ $1.06 \mu \mathrm{m}$ and density $\rho_{p}=1850 \mathrm{~kg} \cdot \mathrm{m}^{-3}$ ). The first column is obtained by fitting the data in steady rotations using a power law with a threshold. The other columns correspond to the angles measured at the end of the relaxations for different initial steady rotation rates.

(velocity strengthening) for all $J$ [Fig. 4(a), grey curve]. Following the measurements, we thus take

$$
\mu(J)=\mu_{0}+\left(J / J_{0}\right)^{\alpha},
$$

with $\mu_{0}=0.104, J_{0}=2.27 \times 10^{-2}$, and $\alpha=0.37$.

For frictional suspensions, experiments suggest that the thickness $h=25 d$ and that the rheology is nonmonotonous with a minimum at a critical viscous number $J_{c}$ [Fig. 8(a), green curve]. Following the measurements, we take

$$
\begin{aligned}
\mu(J) & =\mu_{\text {stop }}+\left[\left(J-J_{c}\right) / J_{0}\right]^{\beta} \quad \text { for } J>J_{c}, \\
J & =0 \quad \text { for } \mu<\mu_{\text {stop }}
\end{aligned}
$$

with $\mu_{\text {stop }}=0.389, \quad J_{c}=10^{-6}, J_{0}=8.69 \times 10^{-3}$, and $\beta=0.7$. When the flow is at rest $(J=0)$, the condition for starting is $\tan \theta=\mu_{\text {start }}=0.51$.

In the following, we numerically solve Eqs. (C2)-(C5) for two cases: (i) a steady rotating drum with a constant rotation rate $\omega$ and (ii) a transient relaxation $(\omega=0)$ after a steady regime of constant $\omega$.

\section{Steady regimes}

Figure 8(b) shows the angle of avalanche obtained numerically from the model for various steady rotation rates within the experimental range. For the frictionless rheology, one recovers that stationary values of the avalanche angle are possible for all values of rotation rates. By contrast, for the frictional rheology, stationary values of $\theta$ are possible only above a critical rotational rate $\omega_{c}$ corresponding to the critical viscous number $J_{c}$. Below this critical rotation rate, the avalanche angle exhibits the characteristic sawtooth shape observed experimentally [compare Fig. 8(b) with Fig. 3(b) of the main text]. In the model, the amplitude of hysteresis $\Delta \theta$ is only set by the difference $\mu_{\text {start }}-\mu_{\text {stop }}$ and is independent of the rotation rate, as in the experiments. Note that in both the frictional and frictionless cases, the stability of the steady solution is a direct consequence of the velocity-strengthening part of the $\mu(J)$ curve, as a linear stability analysis of Eqs. (C2)-(C5) shows. (a)
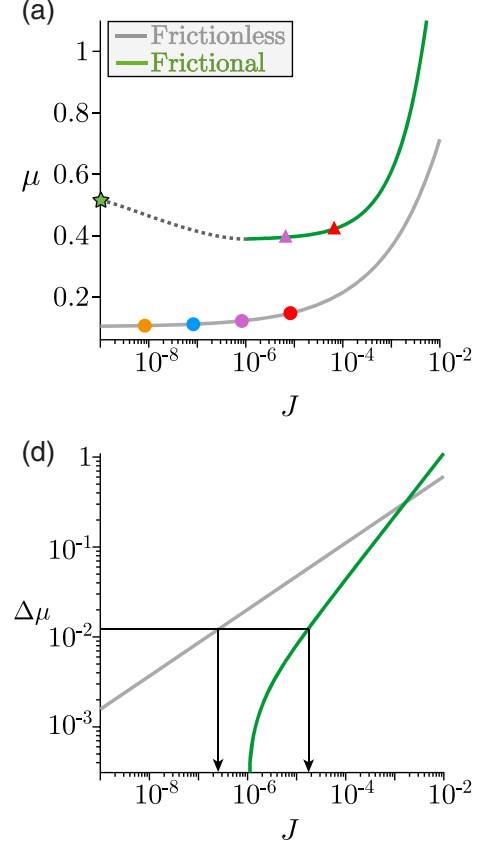

(b)

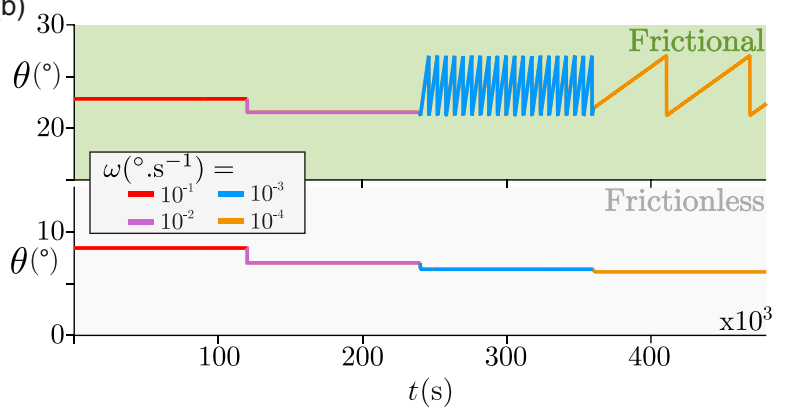

(c)

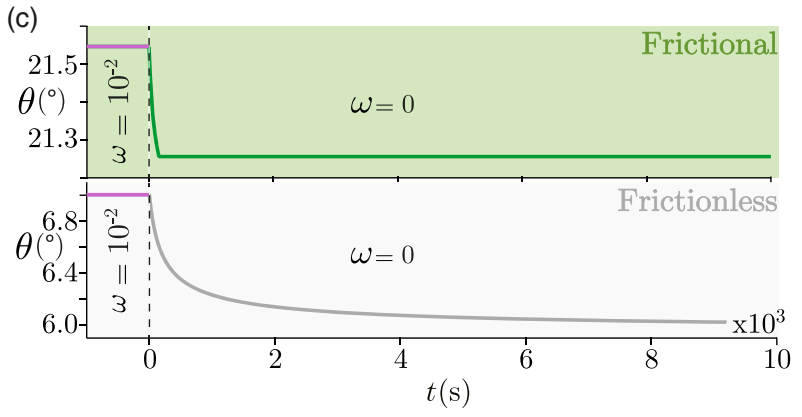

FIG. 8. Prediction of the avalanche model in the rotating drum. (a) Rheologies for the frictionless [grey curve, Eq. (C3)] and frictional [green curve, Eq. (C4)] suspensions as deduced from the experiments. The symbols correspond to the steady states for the constant rotation rates given in panel (b). (b) Time evolution of the avalanche angle for decreasing steps of rotation rates. (c) Relaxation of the avalanche angle when the drum stops $(\omega=0)$ at $t=0$ after a continuous rotation. (d) Reduced macroscopic friction coefficient $\Delta \mu=\mu-\mu_{0}$ versus viscous number $J$ showing that, for a given $\Delta \mu$, the viscous number for the frictional suspension is higher than for the frictionless suspension, thereby explaining the faster relaxations observed for the frictional case. 


\section{Relaxations}

Figure 8(c) shows the relaxation dynamics of the avalanche angle obtained numerically from the model when the drum is stopped after a continuous rotation. Again, the main features of the experimental observations are recovered. For the frictionless rheology, the avalanche angle relaxes asymptotically and smoothly towards equilibrium when $t \rightarrow \infty$. By contrast, for the frictional rheology, the avalanche angle relaxes in finite time with a discontinuity of the time derivative of the angle when the flow stops. These dynamics can be understood by writing Eqs. (C2)-(C5) in the limit of small angle variations $\theta-\theta_{0}$ with $\theta-\theta_{0} \ll \theta_{0}$.

For frictionless grains,

$$
\frac{d\left(\theta-\theta_{0}\right)}{d t} \approx-\frac{J_{0}\left(1+\mu_{0}^{2}\right)^{1 / \alpha}}{\tau}\left(\theta-\theta_{0}\right)^{1 / \alpha}
$$

For frictional grains,

$\frac{d\left(\theta-\theta_{\text {stop }}\right)}{d t} \approx-\frac{1}{\tau}\left[J_{c}+J_{0}\left(1+\mu_{\text {stop }}^{2}\right)^{1 / \beta}\left(\theta-\theta_{\text {stop }}\right)^{1 / \beta}\right]$.

With $\tau^{-1}=\left[\left(h^{3} \Delta \rho g \phi \cos \theta_{0}\right) /\left(\eta R^{2}\right)\right], \quad \theta_{0}=\arctan \left(\mu_{0}\right)$ and $\theta_{\text {stop }}=\arctan \left(\mu_{\text {stop }}\right)$. For frictionless grains, Eq. (C6) implies that $\theta-\theta_{0}$ is strictly positive and tends to zero at infinity with a power law $\theta-\theta_{0} \sim t^{-\alpha /(1-\alpha)}$. For frictional grains, Eq. (C7) implies that $\theta-\theta_{\text {stop }} \sim t^{-\beta /(1-\beta)}$ when $J_{0}\left(1+\mu_{\text {stop }}^{2}\right)\left(\theta-\theta_{\text {stop }}\right)^{1 / \beta} \gg J_{c}$ (initial times) and $\theta-$ $\theta_{\text {stop }} \sim J_{c}\left(t_{f}-t\right) / \tau$ when $J_{0}\left(1+\mu_{\text {stop }}^{2}\right)\left(\theta-\theta_{\text {stop }}\right)^{1 / \beta} \ll$ $J_{c}$. Therefore, relaxation occurs in a finite time $t_{f}$ with a finite $\dot{\theta}=-J_{c} / \tau=-\omega_{c}$. Note that with $\alpha=0.37$ and $\beta=0.7$, the power law of the relaxation for the frictionless grains is smaller than for the frictional grains $(\beta /(1-\beta)>\alpha /(1-\alpha)>0)$. This result implies that the duration of the relaxation is shorter for the frictional grains than for the frictionless grains, as observed experimentally.

[1] B. Andreotti, Y. Forterre, and O. Pouliquen, Granular Media: Between Fluid and Solid (Cambridge University Press, Cambridge, England, 2013).

[2] J. Mewis and N. J. Wagner, Colloidal Suspension Rheology (Cambridge University Press, Cambridge, England, 2012), p. P1.

[3] É. Guazzelli and O. Pouliquen, Rheology of Dense Granular Suspensions, J. Fluid Mech. 852 (2018).

[4] S. Gallier, E. Lemaire, F. Peters, and L. Lobry, Rheology of Sheared Suspensions of Rough Frictional Particles, J. Fluid Mech. 757, 514 (2014).

[5] F. Peters, G. Ghigliotti, S. Gallier, F. Blanc, E. Lemaire, and L. Lobry, Rheology of Non-Brownian Suspensions of Rough Frictional Particles under Shear Reversal: A Numerical Study, J. Rheol. 60, 715 (2016).
[6] F. Boyer, É. Guazzelli, and O. Pouliquen, Unifying Suspension and Granular Rheology, Phys. Rev. Lett. 107, 188301 (2011).

[7] E. DeGiuli, G. Düring, E. Lerner, and M. Wyart, Unified Theory of Inertial Granular Flows and Non-Brownian Suspensions, Phys. Rev. E 91, 062206 (2015).

[8] R. Mari, R. Seto, J. F. Morris, and M. M. Denn, Shear Thickening, Frictionless and Frictional Rheologies in Non-Brownian Suspensions, J. Rheol. 58, 1693 (2014).

[9] M. Wyart and M. E. Cates, Discontinuous Shear Thickening without Inertia in Dense Non-Brownian Suspensions, Phys. Rev. Lett. 112, 098302 (2014).

[10] C. Clavaud, A. Bérut, B. Metzger, and Y. Forterre, Revealing the Frictional Transition in Shear-Thickening Suspensions, Proc. Natl. Acad. Sci. U.S.A. 114, 5147 (2017).

[11] J. Comtet, G. Chatté, A. Niguès, L. Bocquet, A. Siria, and A. Colin, Pairwise Frictional Profile between Particles Determines Discontinuous Shear Thickening Transition in Non-colloidal Suspensions, Nat. Commun. 8, 15633 (2017).

[12] G. Chatté, J. Comtet, A. Niguès, L. Bocquet, A. Siria, G. Ducouret, F. Lequeux, N. Lenoir, G. Ovarlez, and A. Colin, Shear Thinning in Non-Brownian Suspensions, Soft Matter 14, 879 (2018).

[13] B. M. Guy, M. Hermes, and W. C. K. Poon, Towards a Unified Description of the Rheology of Hard-Particle Suspensions, Phys. Rev. Lett. 115, 088304 (2015).

[14] M. Wang and J. F. Brady, Constant Stress and Pressure Rheology of Colloidal Suspensions, Phys. Rev. Lett. 115, 158301 (2015).

[15] Y. Forterre and O. Pouliquen, Flows of Dense Granular Media, Annu. Rev. Fluid Mech. 40, 1 (2008).

[16] M. A. Carrigy, Experiments on the Angles of Repose of Granular Materials 1, Sedimentology 14, 147 (1970).

[17] A. Daerr and S. Douady, Two Types of Avalanche Behaviour in Granular Media, Nature (London) 399, 241 (1999).

[18] O. Pouliquen and Y. Forterre, Friction Law for Dense Granular Flows: Application to the Motion of a Mass Down a Rough Inclined Plane, J. Fluid Mech. 453, 133 (2002).

[19] T. Baumberger and C. Caroli, Solid Friction from Stick-Slip Down to Pinning and Aging, Adv. Phys. 55, 279 (2006).

[20] J. R. Leeman, D. M. Saffer, M. M. Scuderi, and C. Marone, Laboratory Observations of Slow Earthquakes and the Spectrum of Tectonic Fault Slip Modes, Nat. Commun. 7, 11104 (2016).

[21] A. Lucas, A. Mangeney, and J. P. Ampuero, Frictional Velocity-Weakening in Landslides on Earth and on Other Planetary Bodies, Nat. Commun. 5, 3417 (2014).

[22] M. A. Hampton, H. J. Lee, and J. Locat, Submarine Landslides, Rev. Geophys. 34, 33 (1996).

[23] H. J. Lee, Undersea Landslides: Extent and Significance in the Pacific Ocean, an Update, Natural Hazards Earth System Science 5, 877 (2005).

[24] F. Løvholt, S. Bondevik, J. S. Laberg, J. Kim, and N. Boylan, Some Giant Submarine Landslides Do Not Produce Large Tsunamis, Geophys. Res. Lett. 44, 8463 (2017). 
[25] L. Quartier, B. Andreotti, S. Douady, and A. Daerr, Dynamics of a Grain on a Sandpile Model, Phys. Rev. E 62, 8299 (2000).

[26] E. DeGiuli and M. Wyart, Friction Law and Hysteresis in Granular Materials, Proc. Natl. Acad. Sci. U.S.A. 114, 9284 (2017).

[27] S. C. du Pont, P. Gondret, B. Perrin, and M. Rabaud, Granular Avalanches in Fluids, Phys. Rev. Lett. 90, 044301 (2003).

[28] J. R. L. Allen, The Avalanching of Granular Solids on Dune and Similar Slopes, J. Geol. 78, 326 (1970).

[29] H. M. Jaeger, C.-h. Liu, and S. R. Nagel, Relaxation at the Angle of Repose, Phys. Rev. Lett. 62, 40 (1989).

[30] J. Rajchenbach, Flow in Powders: From Discrete Avalanches to Continuous Regime, Phys. Rev. Lett. 65, 2221 (1990).

[31] M. Caponeri, S. Douady, S. Fauve, and C. Laroche, Dynamics of Avalanches in a Rotating Cylinder, in Mobile Particulate Systems (Springer, New York, 1995), pp. 331-366.

[32] P.-E. Peyneau and J.-N. Roux, Frictionless Bead Packs Have Macroscopic Friction, but No Dilatancy, Phys. Rev. E 78, 011307 (2008).

[33] J. N. Israelachvili, Intermolecular and Surface Forces (Academic Press, New York, 2011).

[34] P. Jop, Y. Forterre, and O. Pouliquen, Crucial Role of Sidewalls in Granular Surface Flows: Consequences for the Rheology, J. Fluid Mech. 541, 167 (2005).

[35] G. D. R. MiDi, On Dense Granular Flows, Eur. Phys. J. E 14, 341 (2004).

[36] Note that the friction coefficient $\mu$ is measured at the free surface, while the viscous number $J$ corresponds to an average over the thickness of the flowing layer.

[37] P.-E. Peyneau, Etude du Comportement et du Compactage de Pâtes Granulaires par Simulation Numérique Discrete (Study of the Behavior and Compaction of Granular Pastes by Discrete Numerical Simulations), Doctorate Thesis, Ecole Nationale des Ponts et Chaussées, 2009 (in French).
[38] The asymptotic values $\mu_{0}$ and $\mu_{\text {stop }}$ weakly depend on the rotation rate of preparation before the drum is stopped. Exact values are given in Fig. 7.

[39] P. Olsson and S. Teitel, Critical Scaling of Shearing Rheology at the Jamming Transition of Soft-Core Frictionless Disks, Phys. Rev. E 83, 030302(R) (2011).

[40] E. Lerner, G. Düring, and M. Wyart, A Unified Framework for Non-Brownian Suspension Flows and Soft Amorphous Solids, Proc. Natl. Acad. Sci. U.S.A. 109, 4798 (2012).

[41] R. Fischer, P. Gondret, and M. Rabaud, Transition by Intermittency in Granular Matter: From Discontinuous Avalanches to Continuous Flow, Phys. Rev. Lett. 103, 128002 (2009).

[42] J. A. Dijksman, G. H. Wortel, L. T. H. van Dellen, O. Dauchot, and M. van Hecke, Jamming, Yielding, and Rheology of Weakly Vibrated Granular Media, Phys. Rev. Lett. 107, 108303 (2011).

[43] O. Kuwano, R. Ando, and T. Hatano, Crossover from Negative to Positive Shear Rate Dependence in Granular Friction, Geophys. Res. Lett. 40, 1295 (2013).

[44] A. N. Edwards, S. Viroulet, B. P. Kokelaar, and J. M. N. T. Gray, Formation of Levees, Troughs and Elevated Channels by Avalanches on Erodible Slopes, J. Fluid Mech. 823, 278 (2017).

[45] M. Trulsson, B. Andreotti, and P. Claudin, Transition from the Viscous to Inertial Regime in Dense Suspensions, Phys. Rev. Lett. 109, 118305 (2012).

[46] L. Amarsid, J.-Y. Delenne, P. Mutabaruka, Y. Monerie, F. Perales, and F. Radjai, Viscoinertial Regime of Immersed Granular Flows, Phys. Rev. E 96, 012901 (2017).

[47] M. Trulsson, E. DeGiuli, and M. Wyart, Effect of Friction on Dense Suspension Flows of Hard Particles, Phys. Rev. E 95, 012605 (2017).

[48] R. A. Bagnold, The Shearing and Dilatation of Dry Sand and the "Singing Mechanism", Proc. R. Soc. A 295, 219 (1966). 